\title{
TeraComms: Wireless Communications in the THz Band
}

\section{George Tsoulos* and Georgia Athanasiadou}

University of Peloponnese, Department of Informatics and Telecommunications, Wireless and Mobile Communications Lab, Greece

\section{The Need for THz Wireless Communications}

Frequencies above $6 \mathrm{GHz}$ such as $28 \mathrm{GHz}, 60 \mathrm{GHz}$ have recently gained significant interest for $5 \mathrm{G}$ cellular access with the promised peak data rate around $10 \mathrm{Gbps}$. While this is certainly the way to go, the limited available bandwidth poses constraints on the maximum achievable individual and aggregate data-rates. According to Edholm's law of bandwidth [1], wireless Terabit-per-second (Tbps) links will become a reality by 2020 . This would require modulation and coding strategies with spectral efficiency above $100 \mathrm{bits} / \mathrm{s} / \mathrm{Hz}$ for $7 \mathrm{GHz}$ of bandwidth. Alas this is neither realistic nor has been achieved at lower frequency bands, where the technology is mature. On a similar basis, disk space has increased dramatically and has outgrown the wide-area bandwidth, e.g. 1 TByte stored data requires almost nine days to transfer it over a $10 \mathrm{Mbps}$ wireless connection, i.e. Cloud-based solutions have a very tough time keeping up with storage and computation requirements, and in order to bring the data transfer times down to meaningful levels of a few seconds, Tbps connections are essential. Moreover, projections show [2] that by 2025 , over 7 trillion wireless devices gathering data everywhere and serving over 7 billion people, will lead to a digital society with the ability to know and query anything, anytime, anywhere (in essence an internet of everything - IoE). In the 2030's, similar forecasts predict applications such as brain-to-brain communication, 'Google on the brain', scalable intelligence and downloadable expertise (stream expertise from the internet e.g. to learn a new language, fly an airplane, etc.). Other applications grouped per industrial sector include: a) Media-entertainment, e.g. live relay broadcast of events or disasters, e.g. multi-channel coverage with HDTV (1.5 Gbps), $4 \mathrm{k} / 8 \mathrm{k}$ video ( $>6 \mathrm{Gbps}$ ), live gaming, b) Health, e.g. telemedicine applications with Virtual Reality and Augmented Reality systems (up to $100 \mathrm{Gbps}$ ), c) Transport, e.g. self-driving cars, d) Manufacturing, e.g. remote robot control for factories, e) IT - Networking: Down/ up loading high volume data storage to cloud servers, data center networking, f) Finances, e.g. multiple real time streams for big data killer wireless banking applications, g) Mobile network operators Flash crowd situations, (temporarily huge bandwidth demand from extreme crowdedness or huge number of user devices): Stadium/ conference halls/emergency situations (earthquake, hurricane, road accidents, etc.), h) Last mile services: Enterprise, business or academic users as well as ordinary home users. The requirements for latency below $1 \mathrm{~ms}$ dictate that services must have their contents served from entities physically spaced close to users, most probably placed together with basestations [3]. This is also the case for services that require inter-operator interconnection for low latency services that leads to a substantial increase of CAPEX unless a single network infrastructure shared among operators, is adopted. The single network infrastructure business model can be implemented with the $\mathrm{THz}$ network architecture, hence, leading to a new era of service instead of network competition.

\section{Key Challenges for Wireless Systems in the THz Band}

It is rather obvious that a complete paradigm shift in solid state and radio frequency (RF) technologies as well as in their operation in wireless networks, is required to provide the high rate connectivity

\section{Publication History:}

Received: July 22, 2019

Accepted: December 26, 2019

Published: December 28, 2019

\section{Keywords:}

TeraComms, Wireless communications, Radio frequency, 5G

that is the core lubricant of digital society and its renewal. In this context, wireless communications in the $\mathrm{THz}$ band are quickly gaining momentum as the option to alleviate the capacity limitations of current $4 \mathrm{G}$ as well as $5 \mathrm{G}$ wireless systems and provide a major leap forward towards TeraComms. The $\mathrm{THz}$ band $(0.3-10 \mathrm{THz})$ communication is considered the key technology to enable Tbps links [4-5] mainly due to the huge advantage of the much larger bandwidth, at least an order of magnitude higher than state of the art mm-wave systems, even with multiple windows of tens of $\mathrm{GHz}$ wide each for longer distances. High frequencies result in very small wavelengths $(<1 \mathrm{~mm})$ and hence a large number of antenna elements can be embedded in arrays with very small footprint. This way the very high propagation loss can be compensated for from much higher antenna gains. The absorption from water vapor molecules further increases the pathloss and limits the available bandwidth for distances above several meters, a fact that combined with the very low output power of current $\mathrm{THz}$ transceivers, makes high-gain antennas at both ends of the links an absolute necessity. As in lower frequency communication systems such as IEEE 802.11ac or 4G LTE-A, antenna arrays can be utilized to implement Multiple Input Multiple Output (MIMO), and hence increase either the communication distance by means of beam forming and diversity, or the achievable data rates by means of spatial-beam multiplexing. Much larger antenna arrays with hundreds of elements able to control the radiation signals in 3D or 3D Massive MIMO have also been proposed in order to increase the spectral efficiency. Moving to the $\mathrm{THz}$ band, it will be possible to embed thousands of antennas in not more than $10 \mathrm{~cm} 2$, and hence realize the true potentials of this technique.

Overall, the key challenges for $\mathrm{THz}$ wireless communications could be grouped in four categories:

\section{Channel modeling}

The THz-band channel models have been developed mainly for line-of-sight, and hence there is a clear need for NLOS and multipath modeling [6]. It is crucial to study multipath propagation conditions

"Corresponding Author: Prof. George Tsoulos, University of Peloponnese Department of Informatics and Telecommunications, Wireless and Mobile Communications Lab, Greece; E-mail: gtsoulos@uop.gr

Citation: Tsoulos G, Athanasiadou G (2019) TeraComms: Wireless Communications in the THz Band. Int J Comput Softw Eng 4: 151. doi: https://doi. org/10.15344/2456-4451/2019/151

Copyright: (C) 2019 Tsoulos et al. This is an open-access article distributed under the terms of the Creative Commons Attribution License, which permits unrestricted use, distribution, and reproduction in any medium, provided the original author and source are credited. 
(channel statistics, coherence bandwidth) in order to be able to investigate different system implementations due to the required carrier and sub-carrier spacings, as well as interference statistics. The channel model has to take into account the options of very large arrays in transmission and reception, polarization, as well as the THzband channel propagation effects. All that have to be performed for thousands of parallel channels so that estimation of the available transmission bandwidth is achieved.

\section{Physical layer design}

One of the main challenges at the physical layer is the design of optimal control algorithms able to fully exploit the capabilities of large antenna arrays to maximize the utilization of the THz-band channel. The ability to control the frequency at element basis and the possibility to dynamically create groups of virtual sub-arrays, introduce many degrees of freedom for the design and operation of a massive MIMO communication system. This can be modeled as a resource allocation problem with different optimization goals based on the massive MIMO mode, i.e., dynamic beam forming and spatial multiplexing with multi-band communication. In addition, the unique distancedependent bandwidth provided by the THz-band channel motivates the development of distance-aware adaptive modulation and coding techniques [7] that operate in a single transmission window or over multiple separate bands.

\section{Radio network}

A factor affecting the achievable throughput at the link layer is the impact of multi-user interference. Although the use of very narrow beams in transmission and reception results in very low average interference, these very high gain beams can result into very high values of instantaneous interference, e.g. when they align. The impact of such transient interference needs to be analyzed and mechanisms to overcome it should be designed accordingly. Similarly, the requirement for high-gain antennas simultaneously in transmission and in reception, increases the complexity of broadcasting (need dynamically steered beams at very high speeds while simultaneously transmitting hundreds of Gbps) and relaying (while the unique distance-dependent available bandwidth further motivates the use of shorter links, the overhead associated to beam steering and relay costs, dictates otherwise).

\section{Implementation options}

Possibly the main reason for slow progress in $\mathrm{THz}$ wireless communications is the presence of a unique technical challenge with signal generation, so-called "THz gap" [8], i.e. THz frequencies are too high for the regular oscillators to be efficient and too low for the optical photon emitters to work in. So far, $\mathrm{THz}$ waves are typically generated by either one of these methods, accompanied by a proper frequency multiplier/divider, thus, presenting considerably low output power $(<$ $-10 \mathrm{dBm}$ ). Despite the fact there are some emerging proposals, such as usage of novel type of antennas (e.g. graphene plasmonic antennas) to generate the $\mathrm{THz}$ waves [9], the progress in this field is slow, thus, preventing the rapid expansion of $\mathrm{THz}$ communications. A photonic approach, on the other hand, has a number of advantages. It allows amplitude and phase modulation with high-speed data and recent progress in photonic integration technologies for optical components could enable compact, power-efficient coherent $\mathrm{THz}$ systems with high spectral purity. So the combination of the electrical and optical domains, looks promising, (e.g. with RF over fiber methods).
Implementing optical beam-forming using phased-array antennas reduces the required transmitted power at $\mathrm{THz}$ frequencies. $\mathrm{THz}$ amplifiers with high power, wide bandwidth (and possibly integrated with uni-travelling carrier photodiodes) is another challenge. Fabrication of antenna arrays is also another major issue. For metallic antennas, the main challenge is posed by the design of the array feeding and control network, along with the development of an arrayof-sub-arrays architecture and the balancing between the operations done in the analog or the digital domain. The problem becomes more challenging when metamaterials or nanomaterials are utilized to build plasmonic nano-antenna arrays.

\section{Competing Interests}

The authors declare that they have no competing interests.

\section{References}

1. S. Cherry (2004) Edholm's law of bandwidth. IEEE Spectr 41: 58-60.

2. Diamandis P (2015) Ray Kurzweil's Wildest Prediction: Nanobots Will Plug Our Brains Into the Web by the 2030s. Singularity Hub.

3. 5G PPP (2016) 5G Empowering Vertical Industries. 5G-PPP White Papers.

4. Akyildiz IF, Jornet JM, Han C (2014) Terahertz band: Next frontier for wireless communications. Phys Commun 12: 16-32.

5. Song HJ, Nagatsuma T (2011) Present and future of terahertz communications. IEEE Trans Terahertz Sci Technol 1: 256-263.

6. Han C, Bicen AO, Akyildiz IF (2015) Multi-ray channel modeling and wideband characterization for wireless communications in the terahertz band. IEEE Trans Wirel Commun 14: 2402-2412.

7. Lin C, Li GY (2015) Adaptive beamforming with resource allocation for distance-aware multi-user indoor terahertz communications. IEEE Trans Commun 63: 2985-2995.

8. Petrov V, Moltchanov D, Koucheryavy Y (2016) Applicability assessment of terahertz information showers for next-generation wireless networks. IEEE International Conference on Communications.

9. Jornet JM, Akyildiz IF (2013) Graphene-based Plasmonic Nano-Antenna for Terahertz Band Communication in Nanonetworks. IEEE J Sel areas Commun 31: 685-694. 